\title{
INFLUÊNCIA DO TRATAMENTO TÉRMICO DO RESÍDUO SÓLIDO INDUSTRIAL (GRITS) NA RESISTÊNCIA MECÂNICA DE UM LATOSSOLO PARA PAVIMENTOS DE ESTRADAS FLORESTAIS ${ }^{1}$
}

\author{
Carlos Cardoso Machado², Reginaldo Sérgio Pereira ${ }^{3}$ e José Maurício Machado Pires ${ }^{4}$
}

\begin{abstract}
RESUMO - Em estudos que envolvem o tratamento de solos com aditivos químicos com fins rodoviários, merecem especial importância aquelas pesquisas orientadas no sentido de descobrir novos meios de torná-los mais econômicos e, ao mesmo tempo, mais resistentes. No presente trabalho, o resíduo sólido industrial Grits, oriundo do processo de fabricação de papel e celulose, foi aplicado a um latossolo denominado ETA, característico da microrregião de Viçosa, Minas Gerais, Brasil, com o intuito de melhorar suas características mecânicas para sua aplicação em pavimentos de estradas florestais. O Grits, após receber tratamento térmico a 200, 300, 400, 500 e $600{ }^{\circ} \mathrm{C}$, em mufla, foi misturado no teor de $10 \%$, em peso, ao solo anteriormente citado. Parâmetros geotécnicos, característicos dos ensaios de compactação e resistência à compressão simples, foram utilizados para avaliar o efeito do tratamento térmico nas misturas solo+10\% Grits. Os resultados indicaram que o Grits tem potencial para estabilização de solos de pavimentos de estradas florestais, sendo o melhor resultado alcançado para o Grits tratado a $600{ }^{\circ} \mathrm{C}$, pois houve ganhos de resistência mecânica.
\end{abstract}

Palavras-chaves: Estabilização de solos, resíduo sólido industrial, estrada florestal e tratamento térmico.

\section{INFLUENCE OF THE THERMAL TREATMENT OF INDUSTRIAL SOLID RESIDUE (GRITS) ON THE MECHANICAL RESISTANCE OF A LATOSOL FOR FOREST ENGINEERING ROADS}

\begin{abstract}
Road engineering studies involving chemical stabilization of soils deserve special recognition mainly those directed to lowering the costs of forest engineering roads. This work focuses on soil stabilization of a characteristic Latosol from Viçosa, Minas Gerais, Brazil, herein named ETA, using a residue from paper and cellulose industry production, herein called Grits. Grits content of $10 \%$ related to soil dry weight was used throughout the study after residue thermal treatment using a furnace at 200, 300, 400, 500 and $600{ }^{\circ} \mathrm{C}$. Geotechnical standard parameters from compaction and unconfined compression tests were used to evaluate the effect of thermal treatment on the mechanical response of soil-Grits mixtures. Unconfined compression testing data show that Grits is a promising soil stabilizer, mainly after thermal treatment at $600{ }^{\circ} \mathrm{C}$.
\end{abstract}

Key works: Stabilization of soils, industrial solid residue, forest road, and thermal treatment.

1 Recebido para publicação em 14.11.2002.

Aceito para publicação em 9.9.2003.

Pesquisa financiada pelo CNPq e pela FAPEMIG.

2 Professor Titular do Departamento de Engenharia Florestal da Universidade Federal de Viçosa -DEF/UFV, 36570-000 ViçosaMG; ${ }^{3}$ Doutorando em Ciências Florestais pela UFV e bolsista da CAPES. ${ }^{4}$ Mestre em Geologia pela Universidade Federal de Ouro Preto - UFOP e bolsista do CNPq. 


\section{INTRODUÇÃO}

A falta de solos com características geotécnicas apropriadas, exigidas pelos órgãos rodoviários para a construção de estradas, torna-se um dos grandes entraves para o setor de transportes no Brasil, visto que maiores volumes e maiores cargas são transportados com maior frequiência, a distâncias cada vezes mais longas e problemáticas, exigindo que as estradas, pavimentadas ou vicinais, sejam transitáveis em qualquer época do ano. Salientam-se, também, os custos onerosos envolvidos na sua implantação e manutenção.

Com base em dados de 1995 da Empresa Brasileira de Planejamento de Transportes (Geipot), Bernucci (1997) relata que existe no Brasil 1,5 milhão de quilômetros de estradas de terra (vicinais), sendo as estradas pavimentadas pelos poderes públicos federais, estaduais e municipais, com $148.750 \mathrm{~km}$, equivalentes a apenas 9\% do total da rede. Machado \& Malinovski (1986) estimaram que na década de 80 a extensão da malha brasileira de rodovias florestais era da ordem de 600 mil quilômetros, podendo este número ter aumentado com o decorrer de novas ampliações das empresas florestais.

Todas essas constatações abrem um campo amplo para a busca de materiais alternativos para a construção rodoviária, em particular com um direcionamento aos subprodutos da indústria florestal, visando o emprego destes na estabilização dos solos localizados no subleito e nas margens das estradas florestais e, quando aplicáveis, das vicinais.

No entanto, o interesse pelo emprego de materiais não-tradicionais, entendendo-se como produtos tradicionais o cimento Portland, a cal e os asfaltos artificiais, requer o desenvolvimento de programas extensivos de experimentação de laboratório e campo, de modo a garantir que os novos materiais de construção apresentem os padrões de engenharia exigidos pelas normas técnicas, bem como o desenvolvimento de técnicas construtivas adequadas às características tecnológicas destes novos materiais, do ponto de vista técnico e econômico.

Uma alternativa tecnológica de material para a construção rodoviária e, principalmente, para fins de aplicação em estradas florestais é o Grits, subproduto oriundo do processo de produção da celulose.

O objetivo do trabalho foi analisar, em laboratório, os parâmetros de compactação e resistência à compressão simples para o resíduo sólido industrial Grits, tratado

R. Árvore, Viçosa-MG, v.27, n.4, p.543-550, 2003 termicamente e aplicado a um latossolo característico da microrregião de Viçosa-MG.

\section{REVISÃO DE LITERATURA}

\subsection{Parâmetros Geotécnicos de Interesse Rodoviário}

Machado (1986) e Fernandes (2000) destacaram a granulometria, os limites de Atterberg (limite de liquidez e limite de plasticidade), o equivalente de areia, a compactação, o índice de suporte Califórnia (CBR) e a resistência à compressão simples (RCS) como os parâmetros geotécnicos considerados de suma importância para caracterização geotécnica dos solos para emprego como subleito ou camadas de pavimento de rodovias. Fernandes (2000) destacou, ainda, a permeabilidade como uma propriedade de grande importância das misturas estabilizadas, quando usadas na construção de estradas.

\subsection{Estabilizantes Químicos Tradicionais}

Um material atua como estabilizante químico de um solo, modificando as suas propriedades físicas e químicas, quando, ao ser adicionado a este, reage como agente impermeabilizante, dispersante ou agregante dos materiais presentes neste solo (Lambe \& Michaels, 1954). Para McCarthy (1977), a estabilização química refere-se ao procedimento no qual um material cimentante, ou qualquer material químico, é adicionado ao solo natural, para melhorar uma ou mais de suas propriedades de engenharia. Gidigasu (1976) considera as seguintes variáveis que afetam a estabilização de solos: i) solos: propriedades físicas, químicas e físico-químicas, características da mistura e umidade após a mistura; ii) material estabilizante: quantidade e propriedades; iii) mistura: tipo, método e temperatura; e iv) compactação e cura do produto estabilizante: umidade ótima, método e grau de compactação e cura.

\subsubsection{Histórico}

Este tipo de pesquisa teve seu marco durante a $2^{\text {a }}$ Guerra Mundial, quando a rápida construção de rotas militares era de grande importância. Com poucas exceções, os métodos de estabilização química de solos ainda não podem ser completamente avaliados nos aspectos de física do solo, tecnologia, eficiência e economia, pois existem somente poucos métodos que têm sido comumente aceitos. Perdura, ainda, a necessidade de pesquisas, 
experimentações e observações práticas antes da aceitação generalizada de um determinado método de estabilização (Affleck, 1955; Kédzi, 1979). Nas últimas décadas, tem ocorrido um rápido aumento no uso de estabilizantes químicos; tais aditivos conferem um substancial melhoramento das propriedades de engenharia do solo, quando comparados à estabilização mecânica (Merlim \& Richard, 1982).

São consideradas de uso tradicional, e portanto já padronizadas pelo DNER, apenas as misturas solocimento. A cal hidratada, consagrada internacionalmente, é um dos produtos que podem ser utilizados como material de construção de estradas para melhorar as qualidades de um solo, apesar de não ter seu uso difundido no Brasil, estando ainda na fase de estudo para este fim (Fernandes, 2000). Lima (1981) e Ferraz (1994) relataram que solos estabilizados com a cal apresentaram melhorias nas propriedades elásticas e aumento no CBR e na resistência ao cisalhamento.

\subsection{Aplicação de Resíduos Industriais}

Em 1907, Austim Thomas Byrne discutiu o uso da água do mar e de sais deliqüescentes e, em 1909, o "Bureau of Public Roads" investigou a utilização do licor derivado do processamento de sulfito e do melaço residual de indústria açucareira. $\mathrm{O}$ cloreto de cálcio veio a ser usado na contenção de poeira no leito de ruas de macadame, por volta de 1912 (Hogentogler, 1937).

Estudos de laboratório sobre métodos de alterações das propriedades do solo, com a aplicação proveitosa de diversos resíduos industriais, têm conduzido a muitas propostas para a estabilização de solos (Ingles \& Metcalf, 1977). Um grande número de produtos químicos e subprodutos industriais tem sido experimentado para estabilização dos solos (Singh, 1967). Segundo Sherwood (1961), os resíduos industriais são agentes estabilizantes de baixo custo, pois estão prontamente disponíveis nas áreas onde são produzidos. Obviamente, a distância de transporte pode aumentar o custo desses produtos, tornando o seu uso antieconômico. O autor afirmou, também, que o cloreto de cálcio, o licor negro e as cinzas volantes (fly-ash), estas um resíduo das estações de energia elétrica movidas a carvão mineral, são produzidos em quantidade suficiente para serem aplicados na estabilização.

Segundo Lambe (1955), um obstáculo da expansão do tratamento químico de solos era a pouca comunicação entre as fábricas e os consumidores, e pode-se afirmar que esta situação ainda perdura nos dias de hoje. Na prática comercial, nenhum desses resíduos foi ainda capaz de suplantar em volume de utilização os três principais aditivos: cal, cimento e betume.

Para Ingles \& Metcalf (1977), algumas alternativas de estabilizantes possuem um proveitoso campo de aplicação, em certas circunstâncias especiais, principalmente nos casos em que as soluções temporárias são aceitáveis ou as condições são tais que os altos custos podem ser justificados.

\section{MATERIAL E MÉTODOS}

\subsection{Material}

\subsubsection{O Solo}

O solo estudado é um Latossolo Vermelho-Amarelo, proveniente das imediações da Estação de Tratamento de Água (ETA) da UFV. Este solo é comum nos subleitos das rodovias da região de Viçosa-MG. Localiza-se nos topos de elevações com relevo plano. Apresenta um expressivo horizonte B profundo, poroso e bem drenado, com granulometria argilo-areno-siltosa, podendo ocorrer também como argilo-silto-arenosa. As amostras foram coletadas no terço inferior de um talude de, aproximadamente, $6 \mathrm{~m}$ de altura.

Os critérios utilizados na seleção desse solo foram: I) ele deveria constituir formações representativas e de volumes significativos no universo de solos da região de Viçosa; e II) em seu estado natural, ele não poderia ser empregado como camadas de sub-base de pavimentos rodoviários flexíveis, segundo as recomendações do DER-MG e DNER.

\subsubsection{Resíduo Sólido Industrial Grits}

O Grits é um resíduo sólido industrial gerado no processo de fabricação da celulose. Este resíduo foi tratado termicamente em mufla durante 1 hora, nas temperaturas de $200,300,400,500$ e $600{ }^{\circ} \mathrm{C}$, visando aumentar a sua reatividade. Foi utilizada a porção do material que passou na peneira de malha $2 \mathrm{~mm}$ (número 10 ).

\subsection{Métodos}

\subsubsection{Coleta da Amostra de Solo}

Os procedimentos de campo adotados na coleta da amostra de solo consistiram na extração, no ensacamento

R. Árvore, Viçosa-MG, v.27, n.4, p.543-550, 2003 
e no transporte da amostra para o laboratório de Geotecnia da Universidade Federal de Viçosa. Após esta etapa, a amostra foi seca ao ar e passada na peneira de malha 4,8 mm. Em seguida determinou-se sua umidade hidroscópica e, então, ela foi armazenada em sacos plásticos, para posterior utilização.

\subsubsection{Dosagem}

A dosagem de Grits utilizada, em relação à massa de solo seco, foi de $10 \%$, dosagem esta escolhida com base em ensaios preliminares.

\subsubsection{Ensaios de Laboratório}

Os ensaios realizados com a amostra de solo natural e mistura solo $+10 \%$ Grits, em diferentes tratamentos térmicos, abrangeram as seguintes determinações: a) caracterização - ensaios de granulometria conjunta, limites de Atterberg e massa específica dos grãos de solo; b) análises químicas do solo ETA e do Grits; c) parâmetros ótimos de compactação, na energia do ensaio de compactação Proctor Normal, utilizando o equipamento de Mini-CBR; e d) resistência à compressão simples com moldagem de corpos-de-prova compactados em energia equivalente à do Proctor Normal, na umidade ótima, determinada previamente no ensaio de compactação correspondente à energia do Proctor Normal.

Os corpos-de-prova das misturas, moldados na energia do ensaio de compactação Proctor Normal no teor de umidade Wót, não foram submetidos a um processo de cura, em câmara úmida, antes de serem submetidos aos ensaios de resistência à compressão simples (RCS).

A seqüência de operações para execução dos ensaios de compactação e de compressão simples foram: Solo natural + água - repouso por 1 hora antes da compactação; Solo + 10\% Grits - repouso de 1 hora; em seguida, colocava-se a água - repouso de mais 1 hora antes do início da compactação.

Foram moldados corpos-de-prova com $5 \mathrm{~cm}$ de diâmetro e $5 \mathrm{~cm}$ de altura, para compactação, e $5 \mathrm{~cm}$ de diâmetro e $10 \mathrm{~cm}$ de altura, para a RCS (resistência à compressão simples). Os corpos-de-prova não-curados e não-submetidos à imersão em água foram moldados em triplicata, sendo feita a medição do diâmetro e da altura do corpo-de-prova em três posições; posteriormente fez-se o rompimento em prensa de compressão simples, com velocidade de subida do prato de $1,27 \mathrm{~mm} /$ minuto.

\section{RESULTADOS E DISCUSSÃO}

\subsection{Caracterização e Classificação do Solo ETA}

No Quadro 1 estão os resultados dos ensaios de caracterização geotécnica, bem como a respectiva classificação do solo ETA pelos sistemas HRB (Highway Research Board) e USC (Unifield Soil Classification). Nota-se que esse solo apresenta fração argila elevada (60\%), que é considerada por Ferraz (1997) como a principal origem dos fenômenos físico-químicos que se processam no solo. O valor elevado do LL (limite de liquidez), $75 \%$, pode ser um indicativo de material suscetível ao aumento de volume.

Pelo sistema HRB, o solo ETA se enquadra no grupo A-7-5, com índice de grupo 16, característico de materiais suscetíveis de serem empregados em subleitos de estradas, com um comportamento de fraco a pobre. No Quadro 2 estão os resultados da caracterização química das amostras do solo ETA.

Quadro 1 - Granulometria, limites de Atterberg (LL, LP e IP), peso específico dos sólidos ( $\gamma$ d) e classificação do solo ETA pelo sistema HRB e USC

Table 1 - Granulometry, Atteberg Limits (LL LP and (P), specific solid weigh ( $\gamma d$ ) and ETA soil classification by the HRB and USC system

\begin{tabular}{|c|c|c|c|c|c|c|c|c|c|}
\hline \multicolumn{4}{|c|}{ Granulometria (\%) } & \multicolumn{3}{|c|}{ Limites de Atterberg (\%) } & \multirow{2}{*}{$\begin{array}{c}\gamma \mathrm{d} \\
\left(\mathrm{kN} / \mathrm{m}^{3}\right)\end{array}$} & \multicolumn{2}{|c|}{ Classificação } \\
\hline Argila & Silte & Areia & $\begin{array}{c}\text { Diâmetro } \\
(<0,074 \mathrm{~mm})\end{array}$ & LL & LP & IP & & HRB & USC \\
\hline 60 & 18 & 22 & 78,30 & 75 & 45 & 29 & 27,63 & A-7-5(16) & MH \\
\hline
\end{tabular}

Fonte: Fernandes et al. (2000). 


\subsection{Caracterização Química do Grits}

No Quadro 3 tem-se o resultado da análise química das amostras de Grits. Observa-se que o teor de cálcio suplanta, com exceção da sílica $\left(\mathrm{SiO}_{2}\right)$, os teores dos demais elementos químicos, devendo ser ressaltado que $42 \%$ do cálcio presente no Grits encontra-se na forma de óxido de cálcio $(\mathrm{CaO})$, um dos mais significativos componentes para estabilização de solos.

\subsection{Avaliação do Tratamento Térmico nas Características de Compactação}

Para avaliar a reatividade do solo ETA em presença do Grits a $10 \%$, sem e com tratamento térmico, foi analisado o efeito da adição de Grits nas características de compactação do solo.

Ao analisar o Quadro 4, observa-se que a ação do tratamento térmico do resíduo Grits promoveu uma pequena melhoria nas características de compactação do solo ETA, o que pode ser comprovado pelo decréscimo do teor ótimo de umidade (Wót.) e pelo acréscimo da massa específica seca máxima $\left(\gamma_{\text {dmax }}\right)$,em relação ao solo natural.

Não houve variações significativas no par de valores de teor de umidade ótima e massa específica em relação ao encontrado para a mistura solo $+10 \%$ Grits sem tratamento térmico $\left(28,50 \% \mathrm{e}, 14,32 \mathrm{kN} / \mathrm{m}^{3}\right)$.

\subsection{Avaliação do Efeito do Tratamento Térmico na Resistência à Compressão Simples}

A comparação dos resultados dos ensaios de resistência à compressão simples (RCS), realizados para

Quadro 4 - Parâmetros de compactação do solo natural e das misturas solo+10\% Grits, na energia proctor normal, com o equipamento Mini-CBR

Table 4 - Compactation parameters of natural soil and soil $+10 \%$ Grits mixtures in Proctor Normal energy using the Mini-CBR equipament

\begin{tabular}{|l|c|c|}
\hline \multicolumn{1}{|c|}{ AMOSTRA } & Wót. (\%) & Ydmax. $\left(\mathrm{kN} / \mathrm{m}^{3}\right)$ \\
\hline Solo Natural & 31,80 & 13,53 \\
\hline solo $+10 \%$ grits $\mathbf{S T T} *$ & 28,50 & 14,32 \\
\hline solo $+10 \%$ grits $\mathbf{2 0 0}{ }^{\circ} \mathbf{C}$ & 28,20 & 14,45 \\
\hline solo $+10 \%$ grits $\mathbf{3 0 0}{ }^{\circ} \mathbf{C}$ & 28,40 & 14,41 \\
\hline solo $+10 \%$ grits $\mathbf{4 0 0}^{\circ} \mathbf{C}$ & 29,29 & 14,30 \\
\hline solo $+10 \%$ grits $\mathbf{5 0 0}^{\circ} \mathbf{C}$ & 29,50 & 14,22 \\
\hline solo $+10 \%$ grits $\mathbf{6 0 0}^{\circ} \mathbf{C}$ & 29,10 & 14,34 \\
\hline
\end{tabular}

* STT $=$ sem tratamento térmico.

Quadro 2 - Caracterização química das amostras do solo ETA

Table 2 - Chemical characterization of ETA soil samples

\begin{tabular}{|c|c|c|c|c|c|c|c|c|c|c|c|}
\hline \multicolumn{10}{|c|}{ Elementos - Concentração em \% } \\
\hline $\mathrm{Zn}$ & $\mathrm{Cu}$ & $\mathrm{Mn}$ & $\mathrm{Ca}$ & $\mathrm{Mg}$ & $\mathrm{Fe}$ & $\mathrm{Cd}$ & $\mathrm{Cr}$ & $\mathrm{Pb}$ & $\mathrm{SiO}_{2}$ & $\mathrm{Na}$ & $\mathrm{K}$ \\
\hline 0,004 & 0,007 & 0,072 & 39,50 & 0,091 & 0,81 & $\mathrm{ND}$ & $\mathrm{ND}$ & $\mathrm{ND}$ & 40,0 & 7,0 & 0,8 \\
\hline
\end{tabular}

* ND - abaixo do limite de detecção do equipamento de absorção atômica.

Quadro 3 - Caracterização química das amostras do resíduo industrial Grits

Table 3 - Chemical characterization of industrial residue (Grits) samples

\begin{tabular}{|c|c|c|c|c|c|c|c|c|c|c|c|}
\hline \multicolumn{10}{|c|}{ Elementos - Concentração em \% } \\
\hline $\mathrm{Zn}$ & $\mathrm{Cu}$ & $\mathrm{Mn}$ & $\mathrm{Ca}$ & $\mathrm{Mg}$ & $\mathrm{Fe}$ & $\mathrm{Cd}$ & $\mathrm{Cr}$ & $\mathrm{Pb}$ & $\mathrm{SiO}_{2}$ & $\mathrm{NI}$ & $\mathrm{Al}$ \\
\hline 0,005 & $\mathrm{ND}$ & $\mathrm{ND}$ & 20,0 & $\mathrm{ND}$ & 0,043 & $\mathrm{ND}$ & $\mathrm{ND}$ & $\mathrm{ND}$ & 79,0 & 0,001 & $\mathrm{ND}$ \\
\hline
\end{tabular}

* ND - abaixo do limite de detecção do equipamento de absorção atômica. 
as misturas solo+10\% Grits, encontra-se ilustrada no Quadro 5 e na Figura 1. Os resultados mostraram que houve modificações na resistência mecânica para todos os tratamentos analisados, evidenciando a influência do tratamento térmico no Grits. $\mathrm{O}$ melhor resultado foi obtido para o Grits tratado a $600{ }^{\circ} \mathrm{C}$, pois houve ganhos
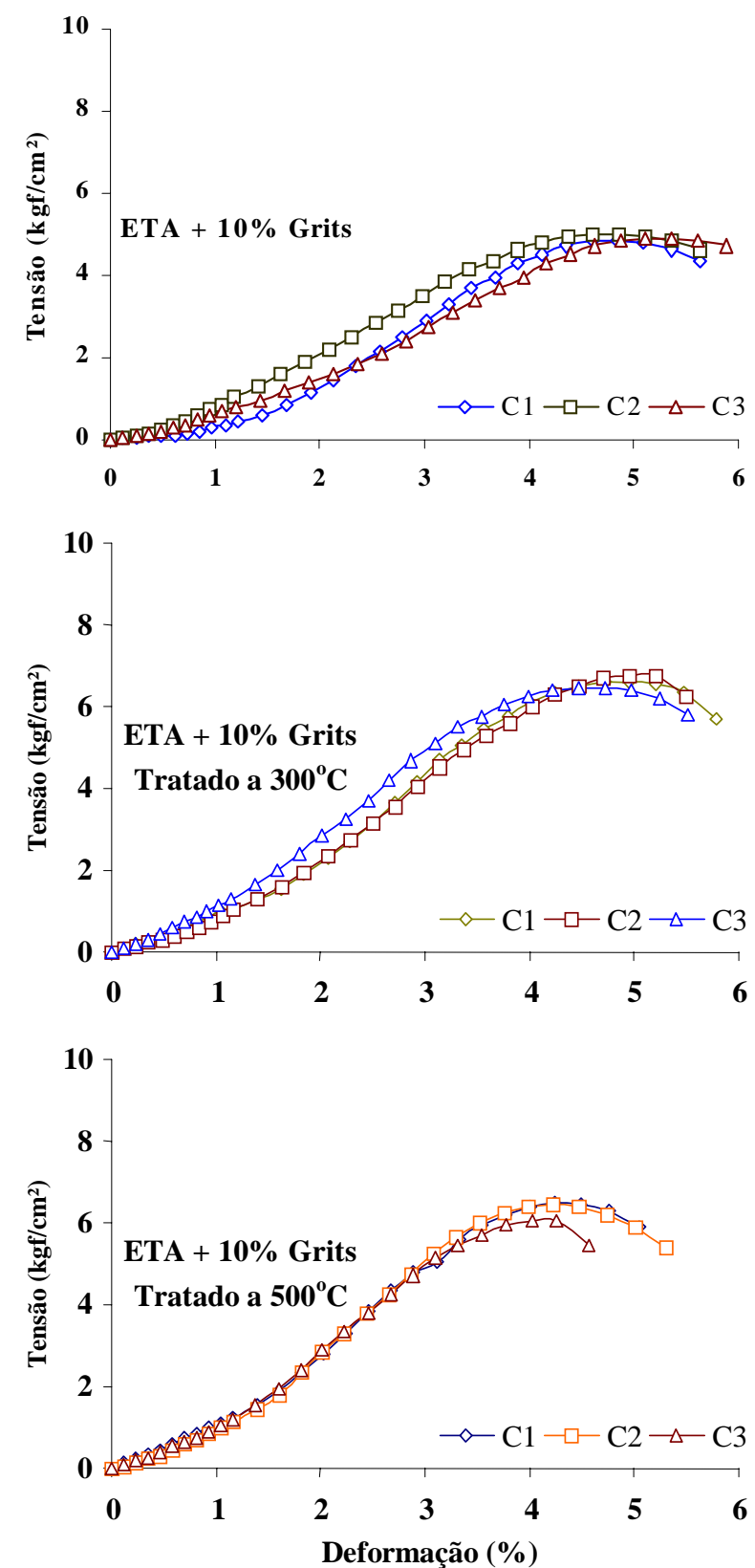

significativos tanto em relação ao solo no seu estado natural como em relação à mistura solo $+10 \%$ Grits sem tratamento térmico. Ressalta-se que não foram aplicados períodos de cura nos corpos-de-prova, o que poderia resultar em maiores ganhos na resistência mecânica das misturas.
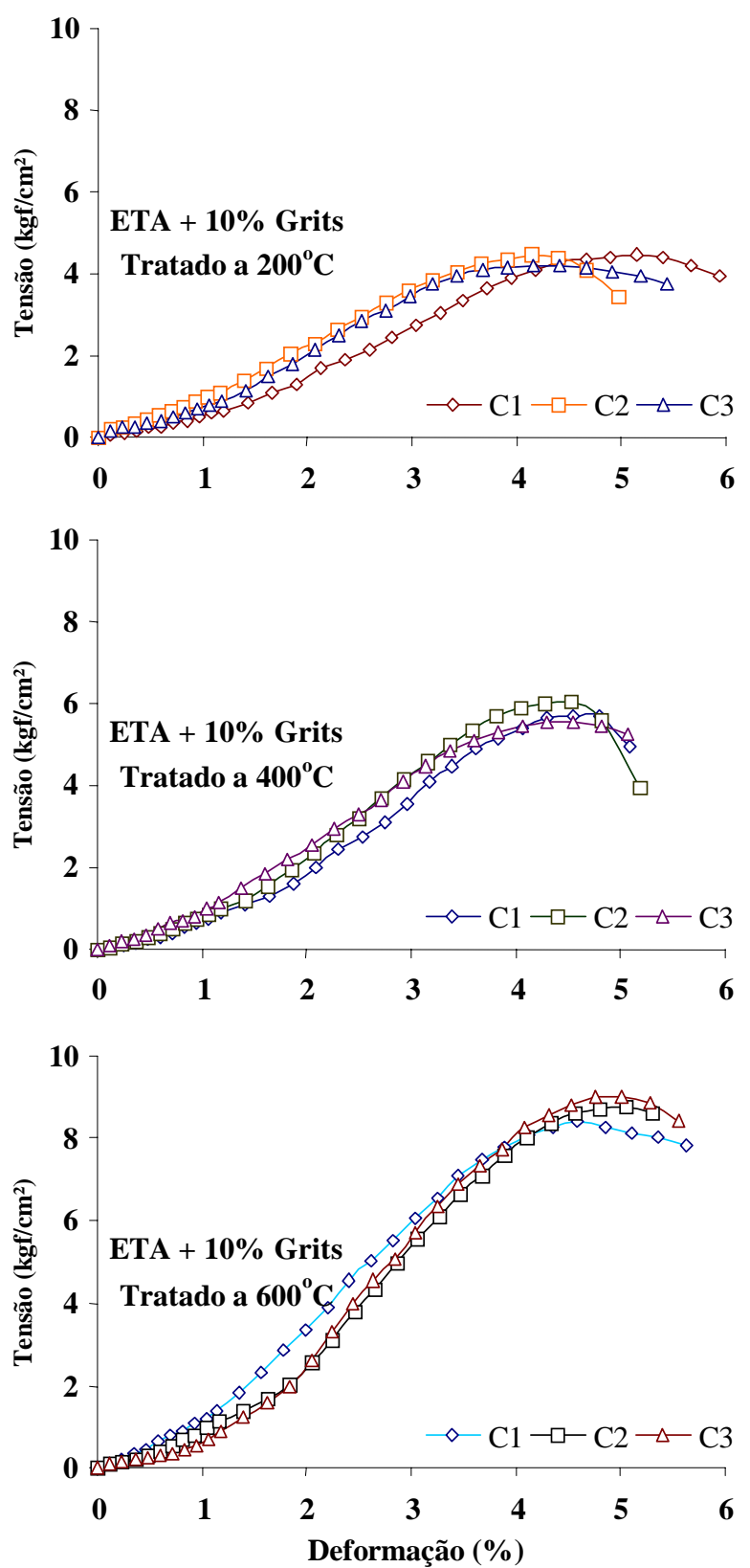

Figura 1 - Curvas tensão x deformação para o solo ETA + 10\% Grits, sem tratamento térmico e tratado nas temperaturas de $200,300,400,500$ e $600^{\circ} \mathrm{C}$, em mufla. As designações C1, C2 e C3 representam os três corpos-de-prova usados no estudo. Figure 1 - Tension $x$ deformation curvs for ETA+10\% Grits soil, without thermal treatment and treated at 200, 300, 400, 500 and $600{ }^{\circ} \mathrm{C}$, in a furnace muffle. The notations $\mathrm{C} 1, \mathrm{C} 2$ and $\mathrm{C} 3$ refer to the three specimens used in the study. 
Quadro 5 - Resistência à compressão simples média, em $\mathrm{KPa}$, para o solo ETA natural e em mistura com o teor de $10 \%$ de Grits, com e sem tratamento térmico, referente à energia Proctor Normal

Table 5 - Average unconfined compressive strength of soil ETA and soil ETA+10\% Grits at the Standard Proctor compaction effort, before and after thermal treatment, in $k P a$

\begin{tabular}{|c|c|c|}
\hline Mistura & $\begin{array}{c}\text { Resistência à } \\
\text { Compressão } \\
\text { Simples } \\
(\mathrm{KPa})\end{array}$ & $\begin{array}{c}\text { Deformação } \\
(\%)\end{array}$ \\
\hline \begin{tabular}{|l} 
Solo Natural \\
Sol Natural
\end{tabular} & 241 & 3,90 \\
\hline solo $+10 \%$ grits STT $*$ & 489 & 5,37 \\
\hline solo $+10 \%$ grits $200{ }^{\circ} \mathrm{C}$ & 445 & 4,65 \\
\hline solo $+10 \%$ grits $\mathbf{3 0 0}{ }^{\circ} \mathbf{C}$ & 659 & 4,88 \\
\hline solo $+10 \%$ grits $400{ }^{\circ} \mathrm{C}$ & 575 & 4,46 \\
\hline solo $+10 \%$ grits $500{ }^{\circ} \mathrm{C}$ & 632 & 4,16 \\
\hline solo $+10 \%$ grits $600^{\circ} \mathbf{C}$ & 872 & 4,88 \\
\hline
\end{tabular}

STT = sem tratamento térmico.

Pela Figura 1 observa-se a variação das propriedades elásticas para as misturas solo-Grits, mostrando a tensão máxima de ruptura dos corpos-de-prova e a correspondente deformação obtida nesse ponto.

\subsection{Comparação do Efeito do Tratamento Térmico nas Misturas Solo-Grits com Valores Obtidos com Outros Aditivos Químicos, Tradicionais e Subprodutos, na Resistência à Compressão Simples (RCS), com Relação ao Solo ETA}

No Quadro 6 estão os resultados de uma comparação entre os aditivos tradicionais e subprodutos e o Grits tratado termicamente a $600{ }^{\circ} \mathrm{C}$, ambos aplicados ao solo ETA, na energia de compactação referente ao Proctor Normal e período de cura igual a zero. Nota-se que o valor obtido para o Grits a $600{ }^{\circ} \mathrm{C}$ suplantou até mesmo os valores obtidos pela cal e pelo cimento, estabilizantes tradicionais, na dosagem de $2 \%$.

Os melhores resultados obtidos por Vieira (1994) e Fernandes (2000), com licor negro kraft e alcatrão de madeira de eucalipto, ambos subprodutos também da indústria florestal, tiveram valores bem inferiores aos obtidos pelo Grits a $600{ }^{\circ} \mathrm{C}$.

\section{CONCLUSÕES}

- O resíduo sólido industrial Grits mostrou-se potencialmente utilizável como agente estabilizante do solo
Quadro 6 - Comparação da resistência à compressão simples, em $\mathrm{KPa}$, entre estabilizantes tradicionais e subprodutos industriais, com o Grits tratado a $600^{\circ} \mathrm{C}$, ambos aplicados ao solo ETA

Table 6 - Comparison of resistance to simple compression, in Kpa, between traditional stabilizers and industrial by-products, with Grits treated at $600{ }^{\circ} \mathrm{C}$, both applied to the ETA soil

\begin{tabular}{|l|c|}
\hline \multicolumn{1}{|c|}{ Mistura } & $\begin{array}{c}\text { Resistência à Compressão } \\
\text { Simples (KPa) }\end{array}$ \\
\hline ETA Natural & 219 \\
\hline Estabilizantes Tradicionais & \\
\hline ETA+2\% Cal* & 356 \\
\hline ETA+2\% Cimento* & 448 \\
\hline Subprodutos Industriais & 282 \\
ETA+2\% Alcatrão (Man-170)* & 199 \\
\hline ETA+0,25\% Licor negro kraft** & \\
\hline ETA+10\% Grits a 600 & \\
\hline ET $\mathbf{C}$ & 872 \\
\hline
\end{tabular}

* Fernandes (2000) e, ** VIEIRA (1994).

pesquisado para pavimentos de estradas florestais.

- Em termos de ganhos de resistência mecânica, o tratamento térmico a $600{ }^{\circ} \mathrm{C}$ foi o mais eficiente.

\section{REFERÊNCIAS BIBLIOGRÁFICAS}

BERNUCCI, L. L. O Módulo resiliente de solos lateríticos e sua aplicação ao dimensionamento de pavimentos de vias de baixo volume de tráfego. In: Simpósio Internacional de Rodovias de Baixo Volume de Tráfego, 1., 1997. Rio de Janeiro. Anais... Rio de Janeiro, 1997. p. 491-508.

BUENO, B. S.; LIMA, D. C. Técnicas de estabilização de solos com fins rodoviários. In: ANAIS DO I SIMPÓSIO BRASILEIRO SOBRE EXPLORAÇÃO E TRANSPORTE FLORESTAL, 1., 1991, Viçosa. Anais... Viçosa: Sociedade de Investigação Florestal, SIF, 1991. p. 143-183.

FERNANDES, D. C. M. Viabilidade do uso de alcatrão de madeira de eucalipto na estabilização de solos residuais para fins rodoviários. 2000. 124 f. Dissertação (Doutorado em Ciência Florestal) - Universidade Federal de Viçosa, Viçosa, 2000.

GIDIGASU, M. D. Laterite soil engineering. Amsterdam: Elsever Scientific Publishing Company, 1976.

HOGENTOGLER, C. A. Engineering properties of soil. New York, McGraw-Hill Book Company, 1937. 434 p.

R. Árvore, Viçosa-MG, v.27, n.4, p.543-550, 2003 
INGLES, O. G.; METCALF, J.B. Soil stabilization principles and practice. S.1., Butterwarhs \& Company, 1977. $374 \mathrm{p}$.

KÉDZI, A. Stabilization earth roads. Amsterdam: Elsevier Scientific, 1979. 327 p.

LAMBE, T. W.; MICHAELS, A. Altering soil properties with chemicals. Chemical Engineering, n ., v. 32, n. 6, p. $488-492,1954$.

LIMA, D. C. Algumas considerações relativas a aspectos da estabilização dos solos, em particular à estabilização solo-cal. 1981. $171 \mathrm{f}$. Dissertação (Mestrado) - Escola de Engenharia de São Carlos, São Carlos, 1981.

MACHADO, C. C.; MALINOVSKI, J. R. Rede viária

florestal. Curitiba: FUPEF, 1986. 157 p.
McCARTHY, D. F. P. E. Essential of soil mechanics and foundations. Virginia: Reston Publishing Company, 1977. $505 \mathrm{p}$.

MERLIM, G. S.; RICHARD, L. H. Soil engineering. 4.ed. São Paulo: Harper \& Row Publishers, 1982. 819 p.

SHERWOOD, P. T. Soil stabilization by the use of chemical admixtures. Roads and Road Constructions, s.1., p. 102-110, 1961.

SINGH, A. Soil engineering in theory and practice. London: Asia Publishing House, 1967. 653 p.

VIEIRA, S. V. Estabilização de solos com licor negro kraft concentrado para fins rodoviários. 1994. $126 \mathrm{f}$. Dissertação (Mestrado em Ciência Florestal) - Universidade Federal de Viçosa, Viçosa, 1994. 